Cahiers de recherches médiévales

Journal of medieval studies

4 | 1997

Être père à la fin du Moyen Âge

\title{
La responsabilité du père dans les sermons du XIIIe siècle
}

Jussi Hanska et Jean-Pierre Dépée

\section{(2) OpenEdition}

Édition électronique

URL : https://journals.openedition.org/crm/968

DOI : $10.4000 / \mathrm{crm} .968$

ISSN : 1955-2424

Éditeur

Honoré Champion

Édition imprimée

Date de publication : 15 décembre 1997

ISSN : 1272-9752

\section{Référence électronique}

Jussi Hanska et Jean-Pierre Dépée, "La responsabilité du père dans les sermons du XIIle siècle », Cahiers de recherches médiévales [En ligne], 4| 1997, mis en ligne le 15 janvier 2007, consulté le 15 décembre 2022. URL : http://journals.openedition.org/crm/968; DOI : https://doi.org/10.4000/crm 968

Ce document a été généré automatiquement le 15 décembre 2022.

Tous droits réservés 


\title{
La responsabilité du père dans les sermons du XIIIe siècle
}

\author{
Jussi Hanska et Jean-Pierre Dépée
}

Le devoir d'élever et d'éduquer les enfants

1 D'après le quatrième commandement du Décalogue, les enfants doivent obéissance et respect à leurs parents afin de vivre longtemps et de réussir dans ce monde. Cela sousentend également l'idée que les parents ont le devoir d'élever leurs enfants en bons chrétiens. Or, au XIII ${ }^{\mathrm{e}}$ siècle, on ne connaissait pas d'enseignement scolaire généralisé, pas même celui des rudiments de catéchisme donné par l'Église. La responsabilité de la socialisation des enfants incombait aux parents et aux parrains, mais en premier lieu aux parents. On considérait que, à partir de sept ans, le devoir d'éducation des garçons appartenait expressément au père et celui des filles à la mère, même si de l'avis de quelques historiens l'enseignement des notions de base du catéchisme revenait à la mère pour les garçons de plus de sept ans également. ${ }^{1}$

2 Les prédicateurs et les auteurs de guides pastoraux tenaient l'éducation des enfants pour une question importante et, dans leurs écrits, il leur fallait souvent traiter tant de sa nécessité que des méthodes utilisées. Ce domaine semblait être plus spécialement celui des membres des ordres mendiants. Dans son article sur ce sujet, Jenny Swanson mentionne trois ouvrages traitant de manière approfondie cette question (Communiloquium de Jean de Galles, Sermones ad status de Guibert de Tournai et De eruditione predicatorum de Humbert de Romans). ${ }^{2}$ On pourrait y ajouter De eruditione filiorum nobiliorum de Vincent de Beauvais.

3 L'apprentissage, qu'il s'agisse de métier, de bonnes manières ou de rudiments de religion, se faisait en règle générale par assimilation des modèles donnés par les plus âgés. Cela apparaît particulièrement bien dans le sermon de Berthold de Ratisbonne sur sainte Élisabeth de Thuringe :

Io. v. c: 'Non potest filius facere nisi quod uideret, etc.'. Ecci x. a: 'Qualis rector ciuitatis, tales habitantes in ea.' Hinc est quod uidemus iuuenes tartharos uiuere pro magna parte ut uixerunt senes tartari ante ipsos accipientes formam uiuendi ab eis, sic gallici ut antecessores eorum, sic theutonici, sic clerici, sic monachi nigri, sic albi, etc. ${ }^{3}$ 
4 Si la force de l'exemple n'était pas suffisante, il fallait appliquer aux enfants des châtiments corporels ou d'autres moyens coercitifs. Au contraire de ce qui est affirmé dans les exposés les plus sombres de l'histoire de l'enfance, les auteurs des ordres mendiants incitaient à respecter une certaine modération dans les punitions corporelles et condamnaient les excès. Il fallait donc utiliser les châtiments corporels, mais pas immodérément. ${ }^{4}$

Si les parents venaient à manquer à leurs devoirs d'éducation ou présentaient carrément des modèles négatifs, les tenait-on pour responsables des péchés de leurs enfants? Dieu punissait-il les pères comme partiellement responsables des péchés de leurs fils? Dans cet article, il sera examiné dans quelle mesure les pères, dans l'esprit de la théologie morale, étaient responsables des péchés de leurs enfants. Notre instrument de travail sera l'exemplum, tiré de l'œuvre du Pseudo-Boèce De disciplina scholarium. Le personnage principal de ce récit est le fils d'un riche romain nommé Lucretius :

...qui tamen clarissimis ortus fuit natalibus, quantitatis procerae et sanquinae qualitatis, mirae siquidem eloquentiae, perspicacis ingenii, sed quotidianis et ultra debitum nuptiis gaudebat, patre poenam deferente, patrimonium enim parentumque census illicite consumebat, cunctisque zelotypis eminebat. Proprio autem adhuc non destitutus pruritu, postea a parentibus ejectus, tandem ab amicis et consortibus destitutus, a creditoribus undique fatigatus, notis et ignotis furum studuit assistere, crucis ab angustiis a patre creberrime redemptus, ultimo tamen parentis pecunia redimi non potuit. Cruci ergo adductus eumdem ad se venire lacrymis compellebat, osculumque voce querula petebat. Pietatis autem motio ad filii petitionem patrem erexit, erectique filius nasum morsu secuit acutissimo dicens: 'Quare a meis primis erroribus incastigatis evasi? Utquid magistri mei documentis non obedivi, sociosque meos contempsi ?' Filii patrem necesse est indulgere castigationi, magistrumque discipuli mores informare.

Ce récit était l'un des exemplum les plus populaires et les plus copiés du XIII ${ }^{\mathrm{e}}$ siècle. ${ }^{6}$ D'ailleurs, ces derniers temps, les récits d'exempla, isolés ou en recueils, ont représenté pour les historiens une source de documents très appréciés. ${ }^{7}$ On sait la place des exempla dans les sermons. Cependant l'utilisation et la diffusion des exempla isolés, ne faisant pas partie de recueils proprement dits, ont été moins étudiées. Dans cet article, on examinera l'exemplum du Pseudo-Boèce cité ci-dessus d'après quelques sources du $\mathrm{XIII}^{\mathrm{e}}$ et du début du XIV ${ }^{\mathrm{e}}$ siècle.

7 Dans les documents utilisés, on pourra remarquer deux tendances manifestes, qu'un choix plus large de sources ne modifierait sans doute pas. Tout d'abord, le récit est naturellement utilisé dans un contexte où il est question des devoirs pédagogiques du père ou, de façon plus large, des parents. Pour la seconde tendance, le contexte, dans lequel apparaitt le récit, est très souvent un commentaire du quatrième commandement du Décalogue.

Péché du fils, père châtié?

8 Les premières apparitions connues du récit du Pseudo-Boèce remontent au début du XIII ${ }^{e}$ siècle. Le moine cistercien Eudes de Cheriton fait mention du récit en 1219 dans son sermon écrit dominica viii ${ }^{a}$ post octauas pentecostes :

Item quidam filium suum, cum paruulus erat, furari et alia illicita sine correctione exercere permisit. Tandem, cum ad uirilem etatem peruenisset, et furtiuo comprensus est, et, cum deberet suspendi, rogauit patrem suum ut daret ei osculum. Cum uero pater ei osculum porrigeret, filius eius cum dentibus frustrum carnis de facie patris rapuit. Cum uero 
inquireretur cur tale enorme comisisset, respondit se merito hoc fecisse, quia pater eius, eo quod prius ipsum corrigere contempserat, ad suspendium perduxit. ${ }^{8}$

9 La version d'Eudes de Cheriton diffère fondamentalement de l'histoire originale du Pseudo-Boèce. Tous les détails se rapportant à la Rome antique ont été supprimés ou bien transformés pour mieux répondre aux conditions du XIII ${ }^{\mathrm{e}}$ siècle. Le fils ne sera pas crucifié, mais pendu. Nous ne savons pas si ces aménagements du récit sont le fait d'Eudes lui-même, ou s'il n'a fait que reprendre une version adaptée d'origine inconnue, car il ne cite pas ses sources. De toute façon, c'est la version d'Eudes qui a circulé pendant tout le Moyen Âge, bien que parfois présentée comme provenant directement de Boèce.

10 Dans le même sermon d'Eudes, on trouve aussi un récit, originaire des Dialogues de Grégoire le Grand, dans lequel un petit garçon blasphème sans en être puni par son père, il finit par mourir de la peste et il voit des Maures qui viennent le chercher pour le conduire en enfer. ${ }^{9}$ Comme nous le verrons, les récits de Grégoire et du Pseudo-Boèce apparaîtront ensemble chez bien des prédicateurs du XIII ${ }^{\mathrm{e}}$ siècle.

11 On retrouve une parution précoce d'un récit du Pseudo-Boèce dans les sermons, avec les Sermones vulgares, écrits dans les années 1228-1240 par Jacques de Vitry. À un détail près, la version de Jacques est semblable à celle d'Eudes. Selon Jacques, le fils n'arrache pas le nez de son père avec les dents, mais il lui mord la lèvre jusqu'au sang. Ce qui est intéressant, c'est que Jacques commence son récit par les mots : «Audivi quod». D'après Claude Brémond, le mot Audivi doit être pris au sens propre. ${ }^{10} \mathrm{Il}$ est manifeste que la version remaniée a circulé oralement dans les milieux cléricaux du début du XIII ${ }^{\mathrm{e}}$ siècle.

12 L'ouvrage d'Étienne de Bourbon Liber de septem donis Spiritus Sancti confirme cette notion de transmission orale. Étienne présente le récit dans une forme connue de l'adaptation d'Eudes de Cheriton et il affirme nettement l'avoir entendue de diverses sources orales:

Audivi a magistro Nicholao de Flaviniaco, archiepiscopo Bisuntinensi, et a pluribus aliis, quod cum quidam pater, sectans tabernas, filium parvulum duceret ibi secum, factus grandis ita assuetus ludis est et tabernis, quod non poterat retrahi, et furabatur primo patri, post vicinis qui ibi expenderet; nec pater suus primo corrigebat eum. Et cum factus esset magnus fur et judicatus duceretur ad suspendium, rogavit pro Deo ut adduceretur ei pater suus; qui cum venisset et fleret, rogavit eum filius ut oscularetur eum et remitteret ei injurias. Quod cum faceret pater, ita momordit eum filius, ut amputaret ei nasum cum labio; et cum de hoc argueretur, ait quod melius debuisset eum occidisse, se potuisset, quia non ipsi bajuli, sed ipse pater eum ad suspendium ducebat, qui eum non corripuerat, sed pocius exemplo suo corrupuerat. Et dicitur quod dimissus ob hoc, vitam suam correxit. ${ }^{11}$

13 La version d'Étienne de Bourbon, comparée aux précédentes, comprend quelques changements importants. Tout d'abord, la responsabilité du père de cet enfant qui est amené à voler est étendue. Dans le récit d'Étienne, non seulement le père ne laisse pas son fils impuni, mais il l'oriente vers le mal par son mauvais exemple. À la manière des récits d'Eudes de Cheriton, celui-ci est modernisé ; on peut egalement mentionner que le fils est conduit à la pendaison par un officier du roi, le bailli (bajulus). L'auteur de l'adaptation du texte semble tenir le père comme unique responsable des péchés du fils. C'est ce qui ressort d'une réplique de celui-ci, dans laquelle il déclare qu'il aurait été juste qu'il tue son père qui, par son exemple, l'avait mené au gibet. La fin du récit va dans le même sens, le fils est gracié et il améliore sa conduite. 
Guibert de Tournai, franciscain français, inclut le récit du Pseudo-Boèce dans son recueil, composé dans les années 1261-1268, Sermones ad status. ${ }^{12}$ Dans le troisième sermon, Ad coniugatas, Guibert cite le Livre de Sirac ( $7: 25:$ « Filii tibi sunt erudi illos.»), d'après lequel il faut éduquer les enfants quand ils sont jeunes, car il est alors facile de les tourner vers le bien. Par contre, il est pratiquement impossible d'enseigner quoi que ce soit aux personnes âgées. Guibert poursuit :

Hic habemus exemplum ex libro Boecii De disciplina scolarium de illo qui cum propter furtum fuit ductus ad suspendium ligatis manibus post tergium uidens patrem suum qui dolens et flens sequebatur eum, et uocans patrem ait: 'Pater da michi osculum.' Et cum oscularetur eum momordit ei nasum dicens : 'Hec omnia mala michi fecisti, qui dum essem puer et te sciente inciperem furari et multa mala facerem non me castigasti. ${ }^{13}$

On sait que Guibert de Tournai a enprunté une grande partie du contenu de ses sermons au recueil de Jacques de Vitry. Celui-ci, dans ses sermons correspondants, insiste sur le fait que l'éducation relève des parents et qu'on est redevable de tout manquement devant Dieu (... aliquin Deo reddituri sunt rationem»). Il n'inclut pas l'exemplum du Pseudo-Boèce dans les sermons destinés aux époux, ${ }^{14}$ mais dans son sermon ad pueros et adolescentes. ${ }^{15}$ Exceptionnellement, Guibert n'utilise pas tant le récit pour démontrer la responsabilité des parents dans les péchés de leurs enfants que pour souligner l'importance de l'apprentissage quand on est jeune.

En ce qui concerne les recueils de modèles de sermons, la démonstration de la diffusion $\mathrm{du}$ récit du Pseudo-Boèce n'est pas aussi facile qu'avec les recueils d'exemplum. Seulement les recueils ad status sont d'utilisation plus aisée, et du XIII ${ }^{\mathrm{e}}$ siècle, il n'en est resté que trois, ceux déjà cités de Jacques de Vitry et de Guibert de Tournai, ainsi que le De eruditione praedicatorum, du dominicain Humbert de Romans, comprenant des ad status où sont utilisés des exempla du Pseudo-Boèce. ${ }^{16}$

17 L'ouvrage Communiloquium de Jean de Galles est une des plus intéressantes sources où paraît le récit du Pseudo-Boèce, dans la partie destinée aux parents des petits enfants. Voici ce qu'il écrit sur les méthodes d'éducation:

...indulgentia approbanda dum caueat peccatum et filiorum dispendium, moderatio laudanda dum teneat ordinatum modum et econtrario negligencia parentum in castigando filios et corripiendo multum uituperanda et peccata ipsorum eis imputanda aliquo modo et pena eis aliquando a Deo inflicta fuit pro eorum negligencia. Sic fuit de Heli qui fuit negligens in corripiendo filios insolentes et immo a Deo merito est punitus $i^{\circ}$ Re. iii ${ }^{\circ}$. Unde narrat Gregorius de Dya. iiii ${ }^{\circ} c a . x v$ de puero quinquenni qui consueuit Deum blasphemare quod fuit percussus a Deo in sinu patris et mortuus et hoc iuste tam in penam patris quia non corripit eum, quam in penam filii ne cresceret malis maioribus crescente etate. Et de hoc narrat Boecius libro De disciplina scolarium de filio Lucrecii sanquineo nomine Zenonis discipulo quod fuit absque disciplina nutritus consumens sua in aleis et meretriciis sicut ibi enumerat et a patre redemptus creberrime de angustiis crucis et deinde captus parentum penuria redimi non potuit. Qui cum lacrimis uoce querula peciit osculum a patre et cum pater accessisset nasum illius morsu acutissimo secuit dicens: 'Quare meis primis erroribus incastigatus euasi ac magistri mei documentis non obediui sociosque meos contempsi? Filii patrem castigatum necesse est indulgere magistrumque discipuli mores informare.' Quasi scilicet dicens patri, quia non castigasti me sed permisisti me uacare peccatis merito de te uindictam capio. Unde et parentes debeant cauere, ne relinquant malum exemplum filiis ut dictum est supra. ${ }^{17}$

18 Dans ce passage, on trouve beaucoup de choses intéressantes. D'abord, Jean écrit timidement que les négligences des parents sont particulièrement blâmables et que les péchés des enfants peuvent être dans une certaine mesure portés sur le compte des 
parents. Il poursuit en disant que Dieu punit parfois les parents du fait de leurs négligences. Il semblerait que Jean ne veuille pas prendre parti de manière catégorique; les parents sont-ils responsables des péchés de leurs enfants lorsque ces péchés résultent d'une éducation négligée? De sûr, il n'avance qu'une chose, les négligences sont grandement critiquables. Selon lui, il est évident que les parents sont responsables dans une certaine mesure des péchés de leurs enfants, mais il ne veut pas prétendre que leur responsabilité est sans ambiguïté.

Les exemples pratiques qu'il a choisis parlent une langue autrement plus claire. Une citation qu'il fait de la Bible (I Rois $3: 13$ ) parle de Samuel à qui le Seigneur apparaît; le Seigneur lui dit maudire la parenté d'Élie car ce dernier n'a pas puni ses fils coupables de péchés. Dans le chapitre suivant, on éloque des combats contre les Philistins pendant lesquels les deux fils d'élie trouvent la mort ; quand on lui annonce la nouvelle, Élie tombe à terre, se rompt la nuque et meurt (I Rois $4: 11-18$ ). Donc Élie meurt et sa famille est maudite parce qu'il n'a pas puni ses fils de leurs mauvaises actions.

Un deuxième exemple vient de l'exemplum des Dialogues de Grégoire le Grand qu'Eudes de Cheriton a fait connaître. Dans la version de Jean de Galles, le petit garçon de cinq ans blasphème le nom de Dieu. Pour le punir, le Seigneur le projette contre la poitrine de son père avec une telle force que l'enfant meurt. Jean fait ressortir la responsabilité du père dans le péché du fils en modifiant le récit de façon que le père également soit puni par ce coup douloureux. Or, dans le récit original de Grégoire, l'enfant meurt de la peste. Il s'agit d'un changement intentionnel, puisque Jean de Galles, dans Communiloquium, mentionne les Dialogues de Grégoire pas moins de vingt-deux fois et toujours directement dans leur forme originale. ${ }^{18}$ La seule explication logique à ce changement, c'est la volonté de Jean de faire ressortir la responsabilité du père pour le péché de son enfant.

21 Et puis, il y a un exemplum du Pseudo-Boèce, dont l'enseignement est le même que dans l'histoire de Grégoire le Grand. Jean cite directement le Pseudo-Boèce, et non pas des documents de seconde main, comme le font bien d'autres auteurs. Il ne modernise pas non plus le texte, mais il le présente fidèlement dans sa version originale. Avec ces récits, Jean de Galles donne à comprendre que les parents portent la responsabilité des péchés de leurs enfants et que Dieu les punira aussi.

Entre les années 1279 et 1292, un exemplum du Pseudo-Boèce paraît sous le titre De filiorum correpcione dans un recueil anonyme anglais appelé Speculum laicorum. Dans ce recueil, le récit est alors généralement diffusé dans une version modernisée. D'après le titre, le message est destiné expressément aux éducateurs, et non aux enfants euxmêmes. Dans ce récit, l'essentiel n'est pas la vie de péché et de délinquance avec la condamnation à mort qui en découle, mais le laisser-aller pédagogique et sa sanction l'amputation du nez. ${ }^{19}$

Par contre, le recueil d'exempla, Fasciculus morum, du début du XIVe siècle, par un auteur franciscain anonyme, a placé le récit sous le titre Humiliandum parentibus. Fasciculus morum rattache le récit du Pseudo-Boèce au quatrième commandement. L'auteur mentionne ce commandement et il cite d'autres passages de la Bible qui le soutiennent convenablement. Puis il déplore que les enfants de l'époque ne respectent pas vraiment leurs parents. Il poursuit: "Et si queritur causa quare liberi sunt ita ingrati, revera responderi potest: propter defectum correctionis et castigationis dum fuerunt iuvenes. » Il illustre l'importance de l'éducation avec deux récits d'exemplum. Si le second relate 
l'histoire du jeune conduit au gibet, le premier est un récit du même genre qui n'a pas paru dans les autres sources:

Unde narratur de quodam divite unicum filium habente, quem tantum fatue dilexit quod pro nullo defectu illum corripere aut castigare voluit. Tale quidem tandem commisit factum quod pater illum redimere non potuit. Ille ergo assumpto patre in fugam et visis virgis in suo itinere tradidit unam patri dicens sibi: 'Plica istam virgam, pater'; et fecit levissime. Tradidit eciam sibi maiorem, et fecit similiter. Cum autem sibi tradidisset maximam virgam et illam plicare non potuisset, dicit patri: 'Sicut, inquit, leviter plicasti tenerem virgam, sic me in iuventute castigare potuisses; sed modo non potes.' Et extracto gladio patrem occidit. Unde Proverbiorum: 'Qui parcit virge odit filium.' Similiter narrat Boicius De disciplina scolarium.....20

Le fait que cette histoire n'ait circulé que dans les manuscrits de Fasciculus morum, et présente de nombreuses similitudes avec le texte de Boèce (père et fils comme personnages, la richesse du père, les crimes du fils, que finalement l'argent ne peut réparer, ainsi que les reproches faits au père et sa part du châtiment), semble indiquer que l'auteur de ce Fasciculus morum a bien composé ce texte lui-même. À quoi peut bien servir un deuxième exemplum si proche du texte de Boèce ? On peut penser que l'utilisation de plusieurs exempla renforçait la valeur de démonstration du récit. Une seconde hypothèse, plus intéressante, est que cela permet de comparer les différences entre les récits.

Dans celui du Pseudo-Boèce, le fils est crucifié pour son crime et le père est amputé du nez en rappel de ses négligences d'éducateur. Dans la nouvelle version de Fasciculus morum, le fils fait une leçon complète à son père (et en même temps au lecteur) sur la façon de se charger de l'éducation des enfants, puis il finit par tuer son père d'un coup d'épée. Dans la nouvelle version, les rôles ont changé ; le fils doit prendre la fuite à cause de ses crimes, mais à la fin de l'histoire, il est toujours en vie ; alors que le père doit payer ses négligences. Le message implicite est que la faute du père est plus grave que les péchés du fils.

Le récit du Boèce a continué à circuler dans les recueils d'exempla au XIV ${ }^{\mathrm{e}}$ siècle. Il paraît dans l'ouvrage de Jean Bromyard Summa praedicantium dans la sous-partie $A b$ infancia; et dans le recueil Alphabetum narrationum du moine dominicain belge Arnold de Liège. Par l'intermédiaire de ce dernier, le récit s'est aussi répandu en Scandinavie, le confesseur de sainte Brigitte, maître Matthias de Linköping l'a copié dans son propre recueil Copia exemplorum, sous le titre Correccio. ${ }^{21}$ La popularité du récit et sa destination sont restées inchangées tout au long du XIV siècle.

Le quatrième commandement et la responsabilité des parents

On a vu précédemment comment le manuel des prédicateurs du XIV ${ }^{e}$ siècle, Fasciculus morum, a rattaché le devoir d'éducation des parents au quatrième commandement du Décalogue. Ce n'était pas un hasard, les œuvres pastorales de la fin du XIII et du début du XIV ${ }^{e}$ siècle font souvent le lien et utilisent aussi dans de nombreux cas des exempla du Pseudo-Boèce. Silvana Vecchio a étudié les occurrences d'éléments du Décalogue dans les sermons du XIII ${ }^{e}$ siècle, et sa liste préliminaire de sermons, dans lesquels sont traités soit l'ensemble des dix commandements soit plus spécialement certains commandements en particulier, est un remarquable instrument de travail pour la recherche dans ce domaine. ${ }^{22}$

Le quatrième commandement et ses commentaires forment un point de départ évident dans l'étude des conceptions de l'Église chrétienne sur l'éducation à n'importe quelle période de l'histoire. Shulamith Shabar a analysé, pour ce précepte, les engagements 
concernant les enfants et des thèmes généraux (humilité, obéissance, comment se soustraire au péché, ainsi que la poursuite des obligations envers ses parents, alors même que l'enfant a atteint l'âge adulte). ${ }^{23}$ Pourtant, elle ne signale pas que le quatrième commandement engage aussi les parents.

Les travaux de cette époque les mieux connus sur le Décalogue sont incontestablement ceux de Bonaventure (Collationes de decem praeceptis, 1267) et de saint Thomas d'Aquin (Collationes in decem praeceptis, 1273). Les Collationes de saint Thomas, notamment, ont été un modèle pour bien des prédicateurs ultérieurs et des rédacteurs de traités sur les Dix Commandements. ${ }^{24}$ Si Bonaventure, dans ses œuvres, n'insiste pas sur les devoirs paternels, il se contente d'en constater le caractère évident : ...pater, habens filium, ipsum instruit et nutritum, et ipsius est filio legare bona sua..$^{25}$

Saint Thomas, lui, explique la signification du quatrième commandement et incite les parents à s'occuper de l'éducation de leurs enfants.

Et debent parentes duo documenta dare filiis, et cito quia « adolescens iuxta uiam suam et cum senuerit non recedet ab ea ", Treni, "Bonum est uiro cum portauerit iugum ", etc. Et sunt illa que docuit Thobias filium suum, scilicet timere Deum et abstinere ab omni peccato....Unde et Deus punit peccatum parentum in filios. ${ }^{26}$

Saint Thomas fait ressortir la responsabilité parentale d'éducation. Et pourtant, dans son recueil, il ne cite pas l'exemplum du jeune homme conduit au gibet. Il n'en sera pas de même avec les dominicains qui, plus tard, présenteront des versions légèrement remaniées du texte de saint Thomas. Le napolitain Hugues de Prato Florido a publié vers la fin du XIII et le début du XIVe siècle une collection de sermons sur la catéchèse, collection qui n'a pas été très populaire puisqu'il ne nous en est resté que neuf exemplaires.$^{27} \mathrm{Ce}$ recueil ne cherchait pas l'originalité, il est composé de textes, écrits sous forme de sermons, d'auteurs antérieurs. Les sermons sur les vertus et les sept péchés capitaux sont adaptés des œuvres de Guillaume Peyraut Summa de virtutibus et Summa de vitiis, et ceux sur le Décalogue sont des adaptations de saint Thomas.

Hugues se conforme au texte de saint Thomas; il mentionne que les enfants doivent respecter leurs parents, parce que c'est d'eux qu'ils ont reçu la vie, la nourriture et l'éducation (uitam, nutrimentum et documentum). Ensuite, il fait remarquer que les parents doivent apprendre à leurs enfants la discipline et les bonnes habitudes. Il appuie ses dires sur deux récits d'exempla que nous avons déjà vus dans d'autres contextes :

Tenentur enim parentes filios suos docere disciplinam Dei et bonos mores. Sed ue illis qui male nutriunt filios. Sunt enim causa mortis eorum et spiritualis et corporalis. Exemplum de ille puero quinquenni de quo Gregorius dicit in Dyalogo, qui mox ut aliud ei animo obsistebat Deum blasphemabat, quam pater corrigere neglexerit. Qui in extremiis laborans ait : 'Obsta pater quia nigri homines ueniunt me tollere.' Et hoc dicitur : 'blasphemauit Deum et mortuus est'. Exemplum de illo qui ibat ad suspendium et petiit patrem suum osculari, et dentibus nasum ei amputauit dicens : 'Quia non correxisti me, modo ad suspendium ducor.' Et propter ea dicitur in Ecclesiastico: 'Qui parcit uirge odit filium suum et qui diligit filium suum assiduat illi flagella. ${ }^{28}$

Hugues de Prado Florido présente le récit de Grégoire le Grand sous sa forme d'origine. Il réussit pourtant à donner au rôle du père un ton plus sombre que Jean de Galles dans son adaptation. Dans sa version, Hugues fait ressortir la gravité du péché paternel du contexte de l'histoire. Il écrit que ceux qui élèvent mal leurs enfants sont responsables de la mort de l'âme et du corps de leurs enfants. L'exemplum tiré des Dialogues de 
Grégoire illustre la culpabilité de la mort spirituelle, alors que l'exemplum du PseudoBoèce sert à prouve que le père est responsable de la mort physique de son fils.

Contemporain d'Hugues, Aldobrandino de Toscanella a écrit, à la fin du XIII ${ }^{\mathrm{e}}$, sous forme de sermons, son propre commentaire du Décalogue, basé sur la collation de saint Thomas d'Aquin. Cet ouvrage d'Aldobrandino n'a jamais été un bestseller de son temps, il n'en est resté jusqu'à nos jours que dix-sept manuscrits. ${ }^{29}$ Aldobrandino reprend l'idée de saint Thomas de la nécessité de l'éducation des enfants, éducation qu'il faut commencer dès le plus jeune âge, et il cite les mêmes arguments de la Bible que saint Thomas. En plus de ceux-ci, il met pourtant en avant l'exemplum du fils conduit au gibet :

Item debemus eos conseruare a puericia per humilitatem, 'quia adolescens iuxta uiam suam etiam cum senuerit non recedet ab ea.' Hic cadit illi qui in suspendio patris nasum momordit quia eum a puericia non correxerat. $\rangle^{30}$

$\mathrm{Du}$ point de vue de l'interprétation, le travail d'Aldobrandino n'apporte rien de nouveau par rapport aux textes étudiés auparavant, mais la façon de présenter l'exemplum est intéressante. Aldobrandino présuppose très nettement que le récit est préalablement connu de ceux à qui il est destiné, il ne ressent pas le besoin de présenter les faits dans leur ensemble, ni de signaler quelle est l'origine du récit. Il suffit de quelques traits caractéristiques pour que le lecteur comprenne de quelle histoire il s'agit. Ces lecteurs à qui s'adressait Aldobrandino se composaient surtout de frères dominicains plus jeunes, et pour les membres de sa congrégation il suffisait donc de citer quelques brefs rappels du récit. Et il faut bien remarquer que ce texte qu'il supposait connu de ses lecteurs n'était pas l'original du Pseudo-Boèce, mais une version adaptée à son temps dans laquelle le fils est pendu au lieu d'être crucifié. ${ }^{31}$

À la fin du XIII siècle au plus tard, l'exemplum du jeune homme conduit au gibet était devenu pour tous les prédicateurs un standard connu que l'on pouvait supposer trouver dans n'importe quel texte traitant en général du quatrième commandement ou de l'éducation des enfants. Il faut bien reconnaître qu'il n'en a pas toujours été ainsi. Le sermon de Berthold de Ratisbonne sur le quatrième commandement ne parle pas du tout de la responsabilité des parents au sujet de l'éducation ou des péchés des enfants. ${ }^{32}$ Jacques de Lausanne, de son côté, mentionne bien la responsabilité des parents en éducation et fait allusion à leur culpabilité en cas de péché (... sic parentes mundani filios sibi obedientes secum trahunt in profundum peccatorum). Il n'utilise pourtant pas l'exemplum du Pseudo-Boèce, pas plus qu'il ne cite explicitement la responsabilité des parents en cas de péché de leurs enfants. ${ }^{33}$

Quel était le péché du père?

La responsabilité du père ou, comme on l'observe le plus souvent, celle des parents dans l'éducation des enfants et au sujet de leurs péchés, n'est pas un thème de recherche historique tout à fait nouveau. Shulamith Shahar aborde cela dans deux passages de son livre Childhood in the Middle Ages. Tout d'abord, elle rappelle que les parents étaient explicitement tenus pour responsables des péchés de leurs enfants jusqu'à la septième année de ces derniers, et elle utilise comme source le guide des confesseurs Summa confessorum écrit au début du XIII ${ }^{e}$ siècle par Thomas de Chobham. ${ }^{34}$ Cependant, elle n'appuie pas son point de vue sur la responsabilité parentale sur un choix plus large de documents, et elle ne mentionne pas les sources utilisées par Thomas de Chobham. Les conceptions de ce dernier apparaîtront également chez des 
auteurs plus tardifs du XIII ${ }^{e}$, en particulier dans l'œuvre de Jean de Galles le Communiloquium:

Immo debent parentes cohibere eos a peccatis quia ut ait Ieronimus epistula LXXXIX usque ad vii annos puerorum peccata parentibus imputantur. ${ }^{35}$

La responsabilité des parents, et plus particulièrement celle des pères -qui étaient les premiers garants de l'éducation de leurs enfants de plus de sept ans- ne se limitait pas aux péchés des enfants de moins de sept ans, les péchés de leurs enfants plus âgés étaient également portés à leur crédit. C'est ce qu'on doit comprendre en lisant entre les lignes le cas du jeune homme conduit au gibet. La faute du père, dans cette affaire, venait de ce qu'il n'avait pas puni son fils pour les péchés que ce dernier avait commis dans sa jeunesse; il s'agissait alors, entre autres, de jeux de hasard et de la fréquentation de prostituées, des péchés bien improbables pour des petits garçons de moins de sept ans.

Dans tous les documents analysés ci-dessus, il est évident que le père doit au moins dans une certaine mesure répondre des péchés de ses enfants. Des divergences de vues apparaissent au sujet du niveau de cette responsabilité. Semble être accepté à l'unanimité le fait que les péchés commis par les enfants de moins de sept ans sont attribués intégralement aux parents, ces enfants de moins de sept ans étant incapables de maîtriser leurs actes et de distinguer le bien du mal. La responsabilité de leurs péchés incombe aux parents à qui revient le contrôle de ce que font leurs enfants mineurs. On considérait que le péché requérait toujours l'expiation, et comme l'enfant ne pouvait pas le faire, cela revenait donc aux parents.

Au-delà de leur septième année, les enfants étaient considérés, dans une certaine mesure, pouvoir répondre de leurs propres actes. Mais on ne les tenait cependant pas encore pour des adultes. Du côté du droit laïc, il est resté de nombreux documents dans lesquels il est signalé que les enfants mineurs ne peuvent pas être punis avec toute la rigueur de la loi. La définition de la minorité a varié en fonction du lieu et du temps, toutefois la majorité était en général fixée pour les filles à 12 ans et pour les garçons à 14 ans. Le même usage semble s'être constitué aussi dans la réflexion des hommes d'Église; les enfants de moins de 14 ans ne se confessaient pas. ${ }^{36}$ Les enfants dont l'âge était compris entre sept ans et l'âge de la majorité étaient donc en partie responsables de leurs péchés, mais une partie de la responsabilité incombait aux parents.

Cette responsabilité parentale ne prenait pas fin, cependant, lorsque l'enfant atteignait sa majorité. Dans les documents d'instruction pastorale, on utilisait l'exemplum du Pseudo-Boèce plus particulièrement pour bien montrer que l'enfant était plus ou moins, encore après avoir atteint l'âge adulte, le résultat de l'éducation de ses parents. Et comme nous l'avons remarqué, on renforçait parfois cette conception en remaniant certains détails du récit original. Cette idée de la responsabilité parentale s'accordait bien avec la conception dominante tirée d'Aristote, selon laquelle le nouveau-né était un esprit vierge, tabula rasa, comme un tableau vide que les parents remplissait par leur éducation. ${ }^{37}$ La personne adulte était donc jusqu'à un âge particulièrement avancé considérée comme le produit de l'éducation de ses propres parents, et, en tant que telle, son éventuel état de pécheur était, aux yeux de Dieu, partiellement la faute des parents. Cependant, il n'apparaissait pas très clairement dans quelle mesure il fallait estimer la punition des parents pour leurs fautes d'éducation, ni comment peser leurs péchés pour négligences. À la lumière des exempla, les sanctions données à cette époque variaient de la douleur physique à la mort. 
Mais quels étaient donc ces péchés des parents qui pouvaient entraîner des sanctions ? Dans les documents étudiés ci-dessus, on en cite deux remarquables, le fait de négliger (negligentia) l'éducation d'une part, et le fait flagrant de donner le mauvais exemple d'autre part. Dans la classification basée sur les sept péchés capitaux, la négligence représente une subdivision du relâchement moral (acedia). Pourtant la Summa de vitiis de Guillaume Peyraut, en traitant de la question de negligentia, ne mentionne pas la négligence dans l'éducation. Et on ne peut ranger le «mauvais exemple» dans aucune classification systématique des péchés.

Il est cependant sûr que les négligences dans l'éducation étaient considérées comme un péché qu'il fallait avouer en confession tout comme les autres péchés. À la fin du XIII ${ }^{\mathrm{e}}$ siècle, Johannes de Fribourg a écrit un guide, tiré de ses propres expériences en la matière et extrêmement populaire auprès de ses frères confesseurs, Confessionale. Dans son introduction, Johannes écrit :

Secundo autem de interrogationibus faciendis circa quasdam personas diuersarum dignitatum, statuum et officiorum instructionem spiritualem tradidi secundum peccata qui a talibus frequentius committitur. ${ }^{38}$

4 Dans le chapitre Ad coniugatos, il présente quels sont les péchés auxquels les époux succombent le plus souvent, et ce que les confesseurs doivent leur demander. En plus des questions les plus ordinaires concernant la légitimité de leur lien conjugal et de leur vie sexuelle, il convient de leur demander s'ils ont négligé l'éducation qu'il faut donner à leurs enfants: Item si filios non nutriuit ad cultum Dei. Malheureusement, Johannes ne dit pas quelles sortes de pénitences une réponse affirmative aurait entraînées; si bien que nous ne pouvons pas savoir avec quelle gravité il jugeait la négligence. De toute façon, il s'agissait d'un problème suffisamment répandu pour que tous les parents se voient posé la question en confession. ${ }^{39}$

Conclusion

45 Les parents avaient le devoir d'élever leurs enfants dans les bonnes manières et en bons chrétiens. De nombreux auteurs de textes pastoraux des $\mathrm{XIII}^{\mathrm{e}}$ et $\mathrm{XIV}^{\mathrm{e}}$ siècles considéraient que ce devoir découlait du quatrième commandement du Décalogue. Pour les petits garçons de plus de sept ans, ce devoir d'éducation appartenait spécialement au père de famille. Les manquements à ce devoir étaient, aux yeux de l'Église, un péché, un péché particulièrement grave dans la mesure où ces négligences conduisaient les enfants à une vie de pécheurs.

Les auteurs de textes pastoraux ont souvent utilisé des récits d'exempla comme moyens pour communiquer à leurs paroissiens leurs devoirs et les conséquences découlant des manquements à ces devoirs. Le récit le plus souvent utilisé dans le cadre de la responsabilité d'éducation paternelle était celui tiré originellement de l'œuvre du Pseudo-Boèce De disciplina scholarium, dans lequel un jeune homme arrache de ses dents le nez de son père parce que ce dernier avait négligé son éducation. Les utilisateurs de ce récit tendent à le modifier de manière à le faire mieux entrer dans le contexte historique du XIII ${ }^{e}$ siècle ; les détails renvoyant à l'histoire et à la culture de Rome sont supprimés et sont remplacés par d'autres convenant à l'époque. Puis au XIV siècle, le récit circule encore dans sa version modifiée, sans qu'il ne soit plus jamais fait mention du document d'origine. Depuis la fin du XIII e siècle, la négligence dans le domaine de l'éducation paternelle est souvent traitée en liaison avec le quatrième commandement du Décalogue. Et celui-ci est compris comme engageant aussi, au-delà de l'enfant, les parents. 
Les auteurs étaient unanimes pour dire que les parents qui, avaient négligé l'éducation de leurs enfants, ou étaient directement responsables de leur perversion pour leur avoir donné le mauvais exemple, portaient aussi une part de responsabilité des péchés commis par leurs enfants. Cette responsabilité ne se limitait pas aux péchés de leurs enfants encore mineurs, mais se poursuivait aussi pendant tout l'âge adulte des enfants. Dans la plupart des cas, la punition des pères accusés de négligence était une douleur physique, parfois la mort. La responsabilité des pères n'était cependant qu'une responsabilité partielle. La peine la plus sévère devait pourtant être purgée, dans la plupart des cas, par l'auteur même du péché. Il y a pourtant eu une exception à cette règle, la version du récit du Pseudo-Boèce écrite par Étienne de Bourbon, dans laquelle le fils a été gracié, le père ayant été reconnu comme véritable coupable.

\section{NOTES}

1. S. Shahar, Childhood in the Middle Ages, Londres et New York 1992. pp. 113, 174. Sur les devoirs des parrains, v. pp. 117-118.

2. J. Swanson, «Childhood and Childrearing in ad status Sermons by Later ThirteenthCentury Friars ", Journal of Medieval History 4/1990, p. 314.

3. Berthold de Ratisbonne OFM, Sermones rusticanus de sanctis. Sermo de beata Elizabet. Uppsala Universitets Bibliotek MS. C 371, f. 31v.

4. J. Swanson, loc.cit., pp.324-329.

5. Pseudo-Boèce, De disciplina clericorum, PL 64, col. 1267.

6. F.C. Tubach, Index exemplorum. A Handbook of Medieval Religious Tales, FF

Communications $\mathrm{N}^{\circ}$ 204, Helsinki 1981, $\mathrm{n}^{\circ} 3488$.

7. Les trois études d'exemplum suivantes sont des standards dans la recherche; les deux dernières comprennent une bibliographie remarquable; $\mathrm{J}$-Th. Welter, L'Exemplum dans la littérature religieuse et didactique du Moyen-Age, Paris 1927 ; C. Brémond, J. Le Goff et J-C. Schmitt, L'«Exemplum », Turnhout 1982 ; J. Berlioz et M.A. Polo de Beaulieu, dir., Les Exempla médiévaux. Introduction à la recherche suivie des tables critiques de l'Index exemplorum de Frederic C. Tubach, Carcassonne 1992.

8. L. Hervieux, Les fabulistes latins. T. 4. Eudes de Cheriton et ses dérives, Paris 1896, pp.19, 316.

9. Voir Grégoire le Grand, Dialogues, IV, 19, SC 265, pp.72-74

10. L'« Exemplum», p. 123.

11. A. Lecoy de la Marche, Anecdotes historiques, légendes et apologues tirés du recueil inédit d'Étienne de Bourbon, dominicain du XIII e siècle, Paris 1877, $\mathrm{n}^{\circ} 43$.

12. S. Gieben, «Rudimentum doctrinae di Gilberto di Tournai con l'edizione del suo Registrum o tavola della materia ", Bonaventuriana. Miscellanea in onore di Jacques Guy Bougerol ofm. A cura di Francisco de Asís Chavero Blanco OFM, vol. 2, Roma 1988, p. 631. 13. Guibert de Tournai OFM, Sermones ad status. Ad coniugatas, sermo tercius, BN. MS. lat. 15943, f. 145v. 
14. D.L. d'Avray \& M. Tauche, «Marriage Sermons in Ad Status Collections of the Central Middle Ages », Modern Questions about Medieval Sermons. Essays on Marriage, Death, History and Sanctity by Nicole Bériou and David d'Avray, Biblioteca di «Medioevo Latino » 11, Spoleto 1994, pp. 88, 121-123.

15. Je dois remercier Madame Carolyn Muessig, pour cette information.

16. S. Shahar, op. cit., p. 172.

17. Jean de Galles OFM, Communiloquium 2.2.1. MS. Vat.lat. 1018, f. 62r.

18. J. Swanson, John of Wales. A study of Works and Ideas of a Thirteenth-Century Friar, Cambridge 1989, pp. 105, 140, 166.

19. Le Speculum laicorum. Édition d'une collection d'exempla, composée en Angleterre à la fin du XIII ${ }^{e}$ siècle. Par J-Th. Welter, Paris 1914, pp. 60-61.

20. Fasciculus morum. A Fourteenth-Century Preacher's Handbook. Édition and Translation by Siegfried Wenzel, Pennsylvania State University Press 1989, pp. 86-90.

21. Magister Matthias Copia exemplorum. Herausgegeben von Lars Wåhlin. Mit Einleitung und Indizes von Margarete Andersson-Schmitt. Studia Seminarii Latini Upsaliensi 2, Uppsala 1990, pp. xi-xii, 24-25, 153.

22. S. Vecchio, «Il decalogo nella predicazione del XIII secolo", Christianesimo Nella Storia 10 (1989), pp. 43-44. Le catalogue de S. Vecchio comprend quelques coquilles. Les sermons sur le Décalogue de Ramon Llull se trouvent dans la partie 76 -et non 176- de la série Corpus Christianorum. Continuatio Mediaevalis. De même, le recueil Sermones de communi sanctorum de Jacques de Lausanne se trouve dans le manuscrit Vat.lat. 1260, et non pas dans le Vat.lat. 1250.

23. S. Shabar, op. cit., pp. 167-168.

24. S. Vecchio, loc. cit., p. 43.

25. Bonaventure, De decem praeceptis collatio V.S. Bonaventurae Opera Omnia. Tomus V, Quaracchi 1891, p. 523.

26. «Les Collationes in decem praeceptis de Saint Thomas d'Aquin. Édition critique avec introduction et notes par Jean-Pierre Torrel ", Revue des sciences philosophiques et théologiques, tome 69 (1985), p. 241.

27. Hugues de Prado Florido a étudié la théologie à Naples dans les années 1288-1289, il est donc raisonnable de penser qu'il a écrit ses sermons après cette période, mais au plus tard en 1322, puisqu'il est mort le 4 décembre de cette année-là. T. Kaeppeli, Scriptores Ordinis Praedicatorum Medii Aevi, volumen II, Rome 1975, pp. 258-260.

28. Hugues de Prato Florido OP, Sermones decalogo preceptorum. Sermo quinto super quartum preceptum, MS. Vat.lat. 4368, f. 128v-129r.

29. S. Vecchio, loc. cit., p. 43 ; T. Kaeppeli, Scriptores Ordinis Praedicatorum Medii Aevi, volumen I, Rome 1970, pp. 40-44.

30. Aldobrandino de Toscanella OP, Expositio decalogi (Sermones 29), MS. Ottob.lat. 1610, ff. $18 \mathrm{r}-\mathrm{v}$.

31. Je n'ai examiné qu'un seul manuscrit de ce texte, de sorte qu'on peut penser que sa brièveté serait plutôt le fait du copiste, et non celui d'Aldobrandino lui-même. Le manuscrit est néanmoins daté de 1322, si bien que, au cas où cet abrégé est dû au copiste, les arguments présentés sont valables. Celui qui a copié le récit au début du $\mathrm{XIV}^{\mathrm{e}}$ siècle a donc considéré cette histoire comme si évidente que cela n'a pas valu la peine de l'écrire in extenso.

32. A.E. Schönbach, «Studien zur Geschichte der altdeutschen Predigt. Die Überlieferung der Werke Bertholds von Regensburg.I », Sitzungsberichte der 
Philosophisch-historischen Klasse der kaiserlichen Akademie der Wissenschaften 151, Wien 1906, pp. 44-47.

33. Jacques de Lausanne OP, Sermones de communi sanctorum. Sermo super quartum praeceptum, MS. Vat.lat. 1260, f. 59r.

34. S. Shahar, op. cit., p.23.

35. Jean de Galles OFM, Communiloquium 3.2.1., MS. Vat.lat. 1018, f.77v. ; J. Swanson, loc. cit., p.317.

36. S.Shahar, loc. cit., p. 25 ; J. Swanson, John of Wales op. cit., p. 128.

37. Ibid. p. 162.

38. Johannes de Fribourg OP, Confessionale, BL.MS. Adit. 19581, f.182v.

39. Ibid., f.191v.

\section{AUTEURS}

JUSSI HANSKA

Université de Tampere Finlande, Département d'Histoire

JEAN-PIERRE DÉPÉE

Centre des Langues - Université de Tampere 